\title{
Research on the Fast and Accurate Estimation Method of Sag Voltage
}

Nghiên cứu phương pháp ước lượng nhanh và chính xác điện áp sụt giảm ngắn hạn

\author{
Truong Ngoc Minh*, Dao Anh Tu \\ Hanoi University of Science and Technology, Hanoi, Vietnam \\ *Email: minh.truongngoc@hust.edu.vn
}

\begin{abstract}
Sag voltage affects the quality of the grid voltage and the working conditions of the electrical equipment. In order to prevent effectively this problem, the authors investigated the estimation method of fast and accurate voltage and phase angle in the presence of sag voltage. The method proposed Least Error Squares (LES) were constructed on the basis of the least squares algorithm and Taylor series expansion. Compared with conventional Clark and Park methods, the LES approach has the following major advantages: faster detection time, reduced deviation of estimated voltage values at the time of failure; faster detection time, reduced deviation of estimated voltage values at the time of failure; being not affected by other fundamental components $50 \mathrm{~Hz}$, the DC components in the grid. The effectiveness of the method is verified by simulation model of a distribution grid in Vinh Phuc province by Matlab / SIMULINK software.
\end{abstract}

Keywords: Sag voltage, least error squares, DVR

\section{Tóm tắt}

Hiện tượng sụt giảm điện áp ngắn hạn ảnh hưởng xấu đến chất lượng điện áp của lưới điện và chế độ làm việc của các thiết bị điện. Để đưa ra biện pháp ngăn ngừa hiệu quả sự cố này, các tác giả nghiên cứu phương pháp ước lượng nhanh và chính xác điện áp và góc pha khi có sụt giảm điện áp ngắn hạn. Phương pháp đề xuất - LES (Least Error Squares) được xây dựng trên cơ sở thuật toán bình phương cực tiểu và khai triển chuỗi Taylor. So với phương pháp truyền thống Clark và Park, thì phương pháp LES có những ưu điểm chính như sau: phát hiện thời điểm sụt áp nhanh hơn; không tăng đột biến giá trị điện áp ước lượng tại thời điểm sự cố; không bị ảnh hưởng của các thành phần khác tần số cơ bản $50 \mathrm{~Hz}$, các thành phần một chiều trong lưới điện. Hiệu quả của phương pháp được kiểm chứng bằng mô hình mô phỏng một lưới điện phân phối tỉnh Vĩnh Phúc bằng phần mềm Matlab/SIMULINK

Từ khóa: Sụt giảm điện áp ngắn hạn, bình phương sai số cực tiểu, DVR

\section{1. Đặt vấn đề}

Sụt giảm điện áp ngắn hạn là hiện tượng gây ảnh hưởng lớn đến chất lượng điện năng trong lưới trung áp bởi tần suất xảy ra lớn, gây hư hỏng hoặc làm giảm tuổi thọ thiết bị dẫn đến những thiệt hại nặng nề. Vì vậy, việc phát hiện nhanh và chính xác sự cố sụt giảm điện áp là yếu tố quan trọng trong nâng cao chất lượng điện áp và phụ tải.

Phương pháp thường dùng hiện nay để phát hiện sụt giảm điện áp sử dụng phép biến đổi Clark và Park. Tuy nhiên, phương pháp này có nhiều nhược điểm như: nhạy cảm bởi với nhiễu điện áp, sóng hài bậc cao; có sai số lớn về điện áp tại thời điểm sự cố... Để khắc phục các hạn chế của phương pháp Clark và Park, trong phần 2 nhóm tác giả nghiên cứu và đề xuất phương pháp LES (Least Error Squares) nhằm mục đích ước lượng điện áp sụt giảm ngắn hạn.

Trong phần 3 bài báo trình bày ứng dụng phương pháp LES cho thuật toán điều khiển với khả năng đáp ứng nhanh, hiệu quả có thể áp dụng cho từng pha riêng biệt, điều này cho phép DVR hoạt động chính xác điện áp sụt giảm trong điều kiện tải tuyến tính và tải phi tuyến. Phần 4 bài báo trình bày một số kết quả mô phỏng một lưới điện phân phối tỉnh Vĩnh Phúc bằng phần mềm Matlab/SIMULINK. Kịch bản mô phỏng là sự cố ngắn mạch 3 pha làm sụt giảm $20 \%$ điện áp danh định khi xem xét các phụ tải khác nhau.

Kết quả bước đầu của bài báo có thể ứng dụng cho các bài toán liên quan đến vận hành lưới điện và có thể sử dụng cho bộ điều khiển của thiết bị khôi phục điện áp động DVR.

\section{Phương pháp phát hiện sụt giảm điện áp ngắn hạn LES}

Dựa trên cơ sở toán học là phương pháp bình phương cục tiểu (Least Squares Method) được giới thiệu trong [1]. Trong các công trình nghiên cứu trước đây [2-4], phương pháp LES được trình bày để $U^{\prime} o^{\prime} c$ luợng tần số và góc pha nhằm phục vụ cho các bài toán bảo vệ và ổn định hệ thống điện. Trong bài báo này, thuật toán LES được phát triển thêm với mục đích Ước luợng điện áp và góc pha, nhằm sử dụng cho các bài toán vận hành lưới điện và ứng dụng cho bộ điều

ISSN 2734-9381

https://doi.org/10.51316/jst.154.etsd.2021.31.5.6

Received: August 24, 2018; accepted: November 2, 2021 
khiển thiết bị khôi phục điện áp động DVR .

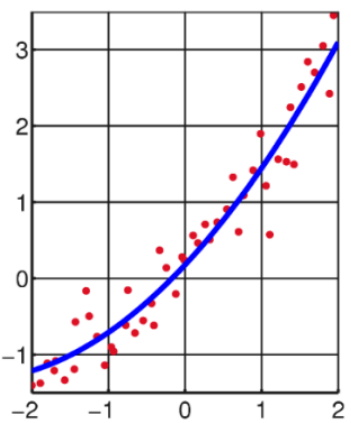

Hình 1. Minh họa phương pháp bình phương cực tiểu

Để ước lượng các tham số điện áp và góc pha sử dụng phương pháp LES, dạng sóng điện áp pha A được giả định là hàm:

$$
u_{a}(t)=k_{a} e^{\frac{-t}{\tau}}+\sum_{n=1}^{\infty} U_{a n} \sin \left(n \omega_{o} t+\theta_{a n}\right)
$$

trong đó : $u_{a}(t)$ : biểu diễn điện áp pha $\mathrm{A}$.

$n$ : đại diện cho các thành phần bậc của sóng hài.

$U_{a n}$ : là biên độ điện áp tương ứng với mỗi thành phần.

$\omega_{0}$ : là tần số góc cơ bản.

$\theta_{a n}:$ là góc pha.

$k_{a}$ : là biên độ của d.c khi $\mathrm{t}=0$.

Theo Taylor, $e^{-t / \tau}$ sẽ được khai triển như sau:

$$
e^{-\frac{t_{1}}{\tau}}=1-\frac{t_{1}}{\tau}+\frac{1}{2 !} \cdot\left(\frac{t_{1}}{\tau}\right)^{2}-\frac{1}{3 !} \cdot\left(\frac{t_{1}}{\tau}\right)^{3}+\ldots .
$$

Sử dụng 3 thành phần khai triển đầu tiên và giả thiết rằng thiết bị điều chỉnh tín hiệu đã chặn được các tín hiệu sóng hài bậc năm và cao hơn bằng các bộ lọc tương tự, thậm chí không có một sóng hài bậc cao nào xuất hiện trong tín hiệu đầu vào, hơn nữa trong thực tế sóng hài ít khi xuất hiện trong trường hợp sự cố về điện áp và dòng điện. Lấy pha $\mathrm{A}$ làm ví dụ, điện áp lấy mẫu tại thời điểm $t_{l}$ có thể biểu diễn phương trình (1) theo công thức rút gọn sau:

$$
\begin{aligned}
v\left(t_{1}\right)= & k_{a}-\frac{k_{a}}{\tau} \cdot t_{1}+\frac{k_{a}}{2 \tau^{2}} t_{1}^{2} \\
& +U_{a 1} \cdot \sin \left(\omega_{0} t_{1}+\theta_{1}\right)+U_{a 3} \sin \left(3 \omega_{0} t_{1}+\theta_{3}\right)
\end{aligned}
$$

trong đó:

$$
\begin{aligned}
& {[A]=\left[\begin{array}{ccccccc}
1 & \sin \left(\omega_{0} t_{1}\right) & \cos \left(\omega_{0} t_{1}\right) & \sin \left(3 \omega_{0} t_{1}\right) & \cos \left(3 \omega_{0} t_{1}\right) & t_{1} & t_{1}^{2} \\
1 & \sin \left(\omega_{0} t_{2}\right) & \cos \left(\omega_{0} t_{2}\right) & \sin \left(3 \omega_{0} t_{2}\right) & \cos \left(3 \omega_{0} t_{2}\right) & t_{2} & t_{2}^{2} \\
\vdots & \vdots & \vdots & \vdots & \vdots & \vdots & \vdots \\
1 & \sin \left(\omega_{0} t_{n}\right) & \cos \left(\omega_{0} t_{n}\right) & \sin \left(3 \omega_{0} t_{n}\right) & \cos \left(3 \omega_{0} t_{n}\right) & t_{n} & t_{n}^{2}
\end{array}\right]} \\
& {[X]=\left[\begin{array}{lllllll}
k_{a} & U_{a 1} \cos \theta_{1} & U_{a 1} \sin \theta_{1} & U_{a 3} \cos \theta_{3} & U_{a 3} \sin \theta_{3} & -\frac{k_{a}}{\tau} & \frac{k_{a}}{2 \tau^{2}}
\end{array}\right]^{T}}
\end{aligned}
$$




$$
\begin{aligned}
& {[A]^{L P I}=\left[[A]^{T} \times[A]\right]^{-1} \times[A]^{T}} \\
& {[V]=\left[\begin{array}{llll}
v\left(t_{1}\right) & v\left(t_{2}\right) & \cdots & \left.v\left(t_{n}\right)\right]^{T}
\end{array}\right.}
\end{aligned}
$$

Biên độ $U_{a 1}$ và góc pha $\theta_{1}$ của thành phần cơ bản sẽ được tính như sau:

$$
\begin{aligned}
& U_{a 1}=\sqrt{X^{2}(2)+X^{2}(3)} \\
& \theta_{1}=\omega_{0}^{\prime} t+\theta_{a 1}=\arctan (X(3) / X(2))
\end{aligned}
$$

Tóm tắt các bước khai triển phương pháp được mô hình hóa trong sơ đồ khối sau:

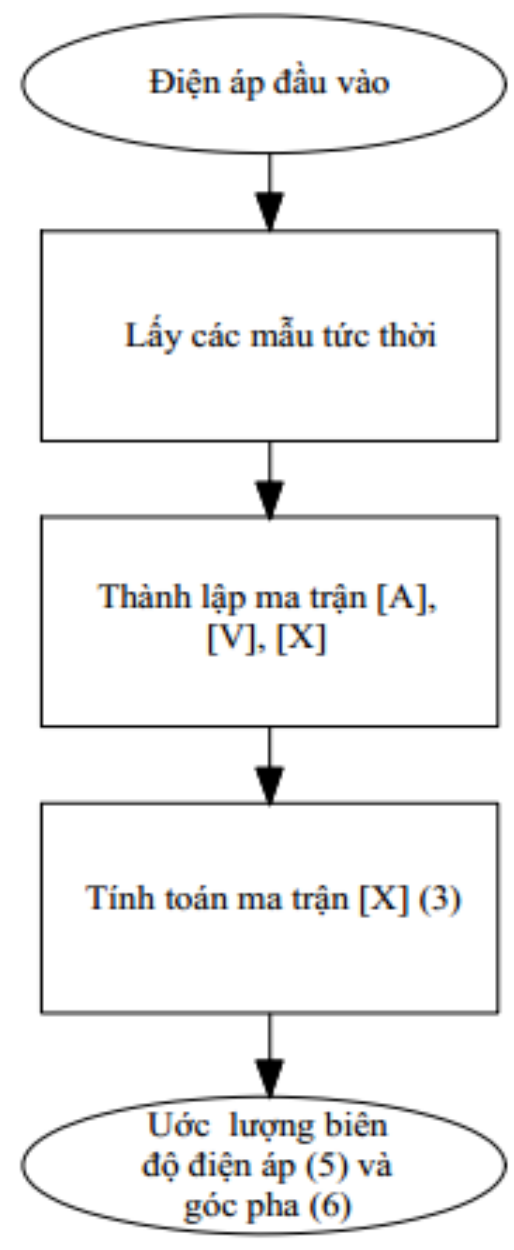

Hình 2. Sơ đồ khối phương pháp LES

\subsection{Các yếu tố ảnh huởng tới kết quả của phuơng pháp LES}

Phương pháp LES bị ảnh hưởng bởi một số yếu tố, chẳng hạn như: số mẫu dữ liệu, tần số lấy mẫu, số lượng thành phần chuỗi Taylor.

\section{Số mẫu dũ liệu.}

Hiệu quả lọc của thuật toán phụ thuộc vào số mẫu dữ liệu. Tuy nhiên nếu số mẫu càng lớn sẽ làm tăng thời gian tính toán và làm giảm tốc độ ước lượng, điều này sẽ làm chậm đáp ứng, tăng thời gian trễ của phương pháp.

Ví dụ, khi tần số lấy mẫu $\left(f_{s}\right)$ là không đổi và sô mẫu $(N)$ là 50 , thì phải mất $2.2 \mathrm{~ms}$ để phương pháp LES hoàn thành. Nhưng khi $N=100$, thời gian hoàn thành trong $4.4 \mathrm{~ms}$.

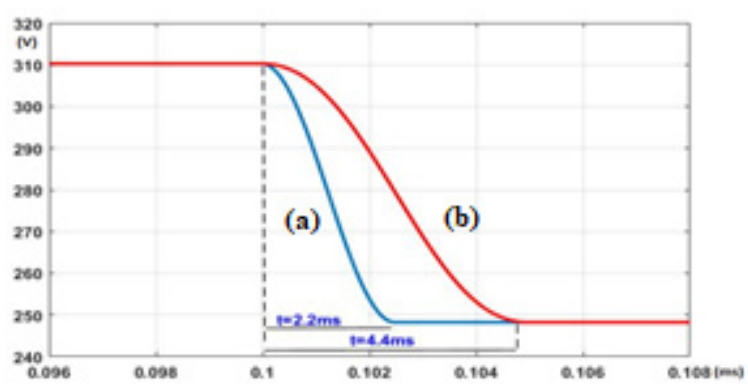

Hình 3. N=50 (đường a) và N=100 (đường b)

Tần số lấy mẫu.

Trong các lý thuyết cho thấy, tần số lấy mẫu cần phải gấp đôi tần số lớn nhất có trong thông tin lấy mẫu để tránh hiệu ứng răng cưa [4]. Trong phương trình (3) thành phần sóng hài bậc cao nhất là $3(150 \mathrm{~Hz})$ nên tần số lấy mẫu nhỏ nhất phải là $300 \mathrm{~Hz}$. Theo nghiên cứu cho thấy giá trị của các phần tử ma trận sẽ giảm khi lượng mẫu lấy được giảm. Hay nói cách khác việc giảm tần số lấy mẫu sẽ làm kết quả đo chính xác hơn nhưng giá trị của các phần tử trong ma trận nghịch đảo lớn hơn, tăng thời gian trễ. Phân tích trên sẽ được chứng minh bằng kết quả hình dưới đây:

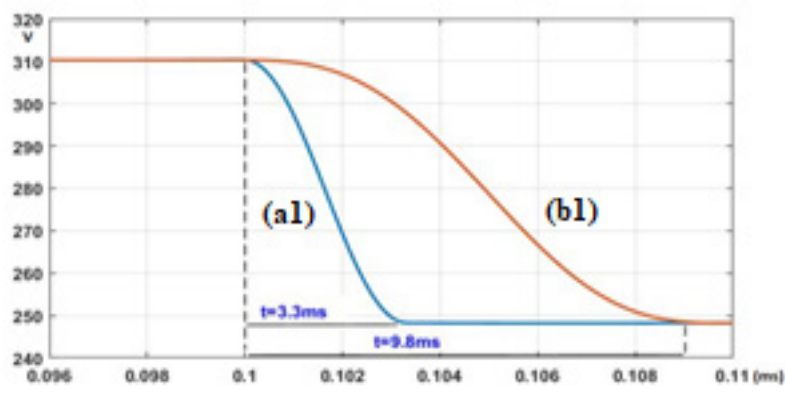

Hình 4. $f=15 \mathrm{kHz}$ (đường a1) và $f=5 \mathrm{kHz}$ (đường b1)

Khi ta tăng tần số lấy mẫu lên, thời gian trễ của phương pháp sẽ giảm đi, nhưng độ chính xác ước lượng sẽ giảm theo. Ví dụ như khi tần số lấy mẫu $f=5 \mathrm{kHz}$ thời gian trễ của phương pháp là $9,8 \mathrm{~ms}$, khi tăng tần số lẫy mẫu lên $f=15 \mathrm{kHz}$ thời gian trê̂ của phương pháp giảm còn $3,3 \mathrm{~ms}$.

\section{Số lượng thành phần chuỗi Taylor.}

Phương pháp được phát triển bằng cách lấy ba phần tử đầu tiên của chuỗi khai triển Taylor của các hàm sin và cos được sử dụng ở trên. Thuật toán cũng có thể mở rộng chuỗi Taylor nhiều hơn như 4 hoặc 5 phần tử. Việc lấy số lượng phẩn tử từ khai triển chuỗi Taylor sẽ quyết định tới độ chính xác cũng như độ trễ của phương pháp. 
Trong bài báo này, các tác giả sử dụng 3 phần tử đầu tiên từ chuỗi khai triển. Ví dụ như khi lấy 3 phần tử đầu tiên của chuỗi thời gian trễ của phương pháp là $3,3 \mathrm{~ms}$, khi lấy 4 phần tử đầu tiên của chuỗi thời gian trễ của thuật toán tăng lên $5,2 \mathrm{~ms}$

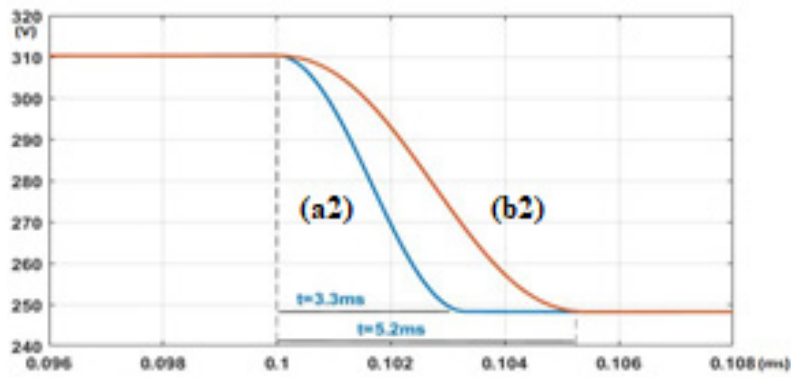

Hình 5 . Lấy 3 phẩn tử (đường a2) và khi lấy 4 phần tử (đường b2)

2.2. Mô phỏng bộ LES filter sử dụng phần mềm Matlab-Simulink

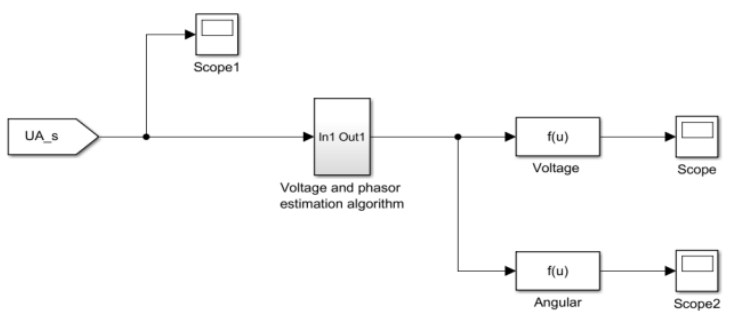

Hình 6. Mô hình mô phỏng bộ LES filer

Khối tín hiện U_s: là tín hiệu điện áp đầu vào được lấy từ điểm đo.

Khối Voltage and phasor estimation algorithm xử lý tín hiệu sử dụng phương trình (2).

Khối Voltage sử dụng phương trình (6) xuất ra điện áp đầu ra.

Khối Angular sử dụng phương trình (7) xuất ra tín hiệu góc phase.

Vi du 1 :Xét trường hợp khi có sự cố ba pha khi nguồn có sóng hài bậc 5 và bậc 7 trong khoảng thời gian từ $0,05 \mathrm{~s}$ đến $0,2 \mathrm{~s}$.

Trong ví dụ này, ta thấy khi nguồn có sóng hài bậc cao, làm cho tín hiệu điện áp không còn sin nữa, gây méo điện áp. Lúc này trên tọa độ $d q$ phương pháp Clarck-Park xuất hiện nhưng dao động, các nhiễu lớn. Còn với phương pháp LES biên độ điện áp của thành phần tần số cơ bản được ước lượng, không chịu ảnh hưởng bởi sóng hài bậc cao

Vi du 2: Xét trường hợp sự cố thay đổi điện áp 1 pha (pha $\mathrm{A}$ ) trong khoảng từ $0,1 \mathrm{~s}$ đến $0,15 \mathrm{~s}$ xảy ra sụt áp pha $\mathrm{A}$ xuống 0,8 pu. Từ $0,15 \mathrm{~s}$ đến $0,2 \mathrm{~s}$ xảy ra tăng áp pha A lên 1,2 pu.

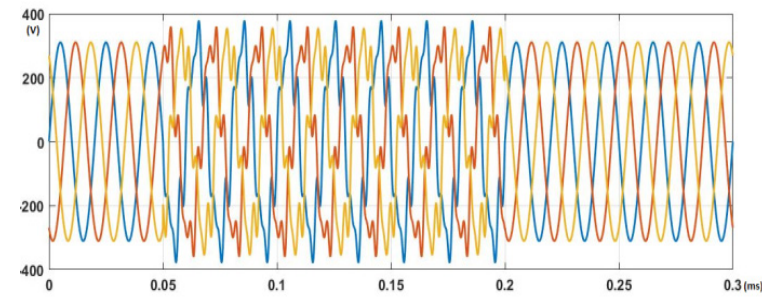

a. Điện áp trên hệ tọa độ ba pha $\mathrm{ABC}$

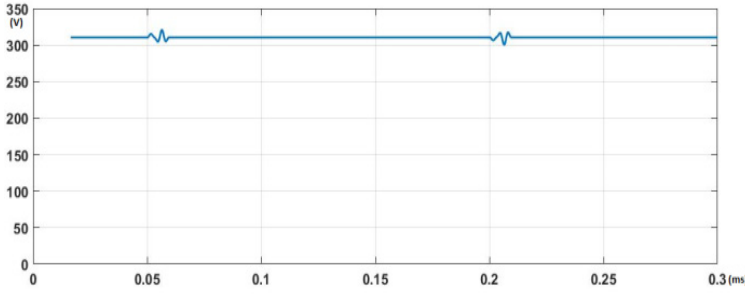

b. Phương pháp sử dụng LES

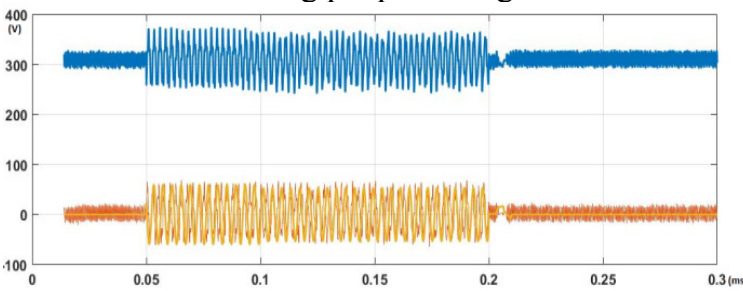

c. Phương pháp sử dụng Clark và Park

Hình 7.

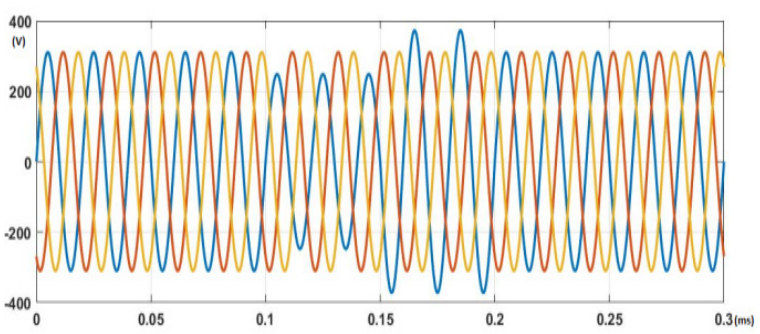

a. Điện áp trên hệ tọa độ ba pha $\mathrm{ABC}$

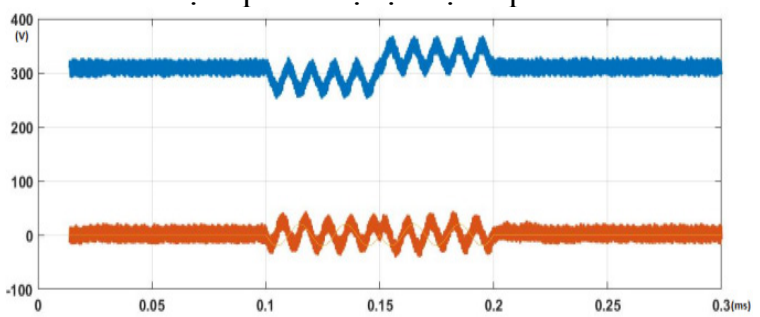

b. Phương pháp Clark và Park

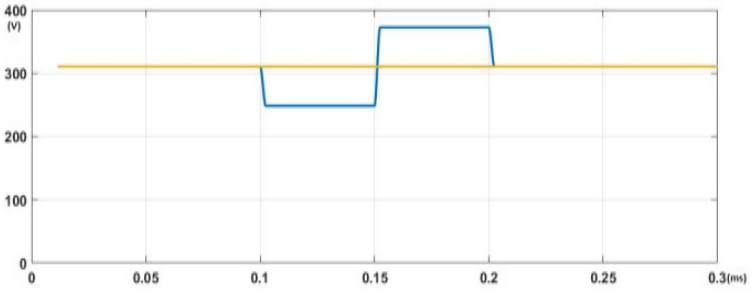

c. Phương pháp sử dụng LES

Hình 8. 
Tại thời điểm xảy ra sự cố $0,1 \mathrm{~s}$ :

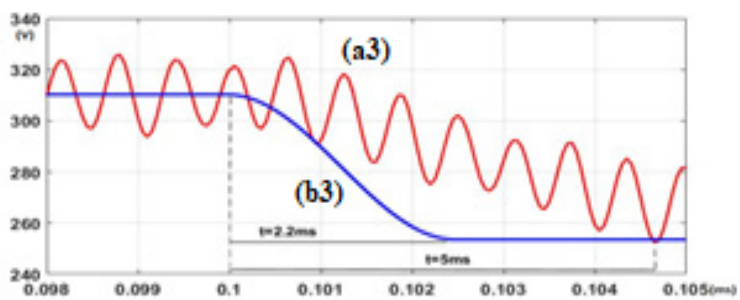

Hình 9. Phương pháp Clark và Park (đường a3) Phương pháp LES (đường $b 3$ )

Ta có thể thấy thời gian phát hiện sự cố phương pháp LES nhanh hơn gấp 2 lần $(2,2 \mathrm{~ms}<5 \mathrm{~ms})$ so với phương pháp Clark và Park

Vi du 3 :Trường hợp ngắn mạch 3 pha trong khoảng $0,1 \mathrm{~s}$ đến $0,2 \mathrm{~s}$ xảy ra sụt áp xuống 0,8 pu.

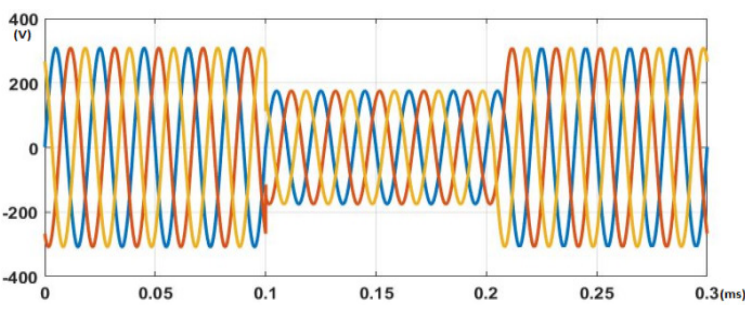

a. Điện áp trên hệ tọa độ ba pha $\mathrm{ABC}$

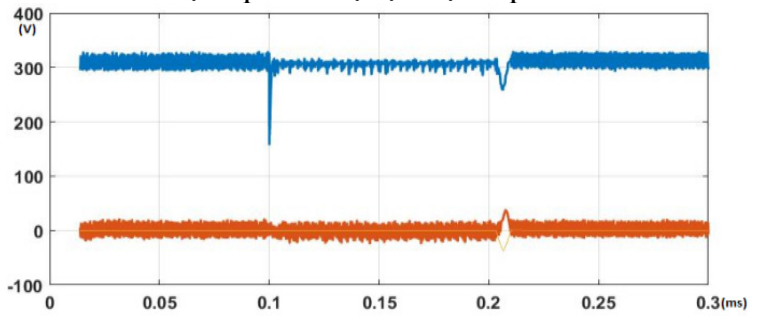

b. Phương pháp Clark và Park

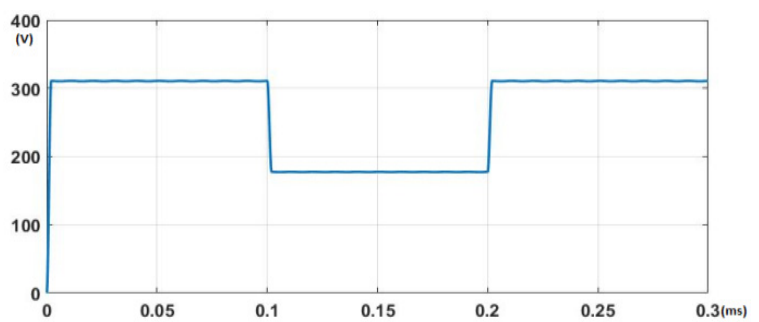

c. Phương pháp sử dụng LES.

Hình 10

Qua ví dụ trên ta thấy, điện áp ước lượng được của phương pháp sử dụng LES đã giảm thiếu tối đa hiện tượng sai lệch điện áp tại thời điểm xảy ra sự cố (giá trị sai lệch $3 \mathrm{~V}$ ), trong khi đó khi sử dụng phương pháp Clark và Park điện áp sai lệch lên đến $150 \mathrm{~V}$.

Nhận xét chung: Các kết quả mô phỏng cho thấy các ưu điểm của thuật toán LES so với phương pháp thường dùng Clark-Park như: giảm thiểu sai lệch điện áp bù tại thời điểm sự cố, thời gian phát hiện sụt giảm điện áp nhanh, cũng như không chịu ảnh hưởng bởi các thành phần tần số khác cơ bản, nhiễu điện áp và các thành phần $\mathrm{DC}$ trong lưới điện.

\section{3. Ứng dụng phương pháp LES cho bộ điều khiển thiết bị DVR.}

Một phương pháp điều khiển nhanh chóng, chính xác và hiệu quả được trình bày trong [5],[6] khi kết hợp sử dụng phương pháp LES để ước lượng độ lớn và pha của điện áp đo được, sẽ làm giảm đi đáng kể sự tác động của các nhiễu, sóng hài... Điều này cho phép DVR phát hiện và khôi phục chính xác điện áp sụt giảm trong điều kiện tải tuyến tính và tải phi tuyển. Phương pháp điều khiển không cần sử dụng bất kỳ vòng khóa pha (PLL) nào. Bên cạnh đó việc kiểm soát riêng rẽ điện áp từng pha cho phép DVR điều chỉnh được các thành phần thứ tự không, thứ tự nghịch của điện áp tải.

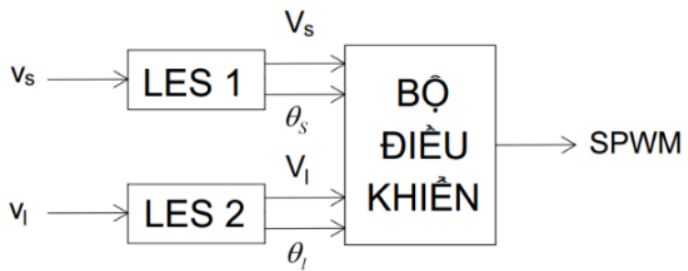

Hình 11. Bộ thống điều khiển DVR cho từng pha

Sơ đồ khối của hệ thống điều khiển DVR được áp dụng cho từng pha riêng biệt, giả sử các thành phần tần số cơ bản và điện áp pha của tải đã biết và được biểu diễn dưới dạng:

$$
\begin{aligned}
& v_{s}=V_{s} \times \cos \left(\omega t+\theta_{s}\right) \\
& v_{l}=V_{l} \times \cos \left(\omega t+\theta_{l}\right)
\end{aligned}
$$

Sử dụng 2 bộ LES như nhau để đo biên độ điện áp và góc pha của 2 tín hiệu $v_{s}$ và $v_{l}$, được biểu diễn dưới dạng $\vec{V}_{s}=V_{s} \angle \theta_{s}$ và $\vec{V}_{l}=V_{l} \angle \theta_{l}$. Phương pháp điều khiển có chức năng ghi lại pha của điện áp bước trước của nguồn cấp để sử dụng làm góc pha tham chiếu cho điện áp tải. Bộ điều khiển DVR được cài đặt cho phép thiết bị hoạt động bù khi điện áp sụt giảm dưới 0,95 pu của điện áp danh định (trong quá trình sụt giảm điện áp). Bộ điều khiển sẽ khôi phục điện áp thành phần tần số cơ bản lại đúng bằng điện áp sụt giảm. Nguyên lý làm việc của bộ điều khiển được mô hình hóa như sau :

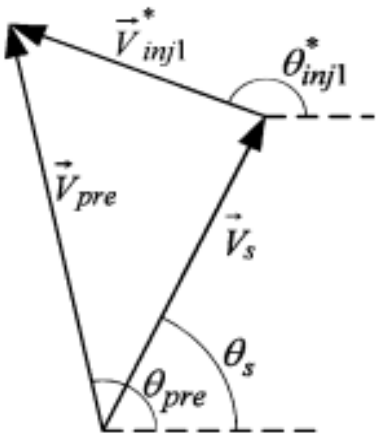

Hình 12. Sơ đồ tính toán vector điện áp bù của DVR 


\section{Một số kết quả tính toán}

Mục đích kết quả mô phỏng là so sánh hiệu quả hoạt của thiết bị khôi phục điện áp DVR khi ứng dụng 2 phương pháp phát hiện sụt giảm điện áp là LES và Clark-Park. Dựa trên số liệu xuất tuyến 474E25.5 của lưới điện trung thế tỉnh Vĩnh Phúc, dựa trên số liệu thực tế của lưới điện trung thế tỉnh Vĩnh Phúc, xuất tuyến 474E25.5 đã được xây dựng. Xuất tuyến 474E25.5 là một trong những xuất tuyến $22 \mathrm{kV}$, cung cấp điện cho các phụ tải từ trạm biến áp $110 \mathrm{kV}$ Vĩnh Tường. Sơ đồ được xây dựng từ các thông số của phụ tải và đường dây từ cột 1 đến cột 208 . Tại vị trí cột 162 rẽ nhánh cung cấp điện cho phụ tải 1 và 2 với công suất mỗi phụ tải là $2,27 \mathrm{kVA}$ qua trạm biến áp, khoảng cách $0.994 \mathrm{~km}$ bằng dây $\mathrm{AC} 50$, nhóm tác gỉa thực hiện mô phỏng trường hợp sự cố ngắn mạch 3 pha trên 2 loại tải chính trong hệ thống điện là tải tuyến tính và tải phi tuyến.

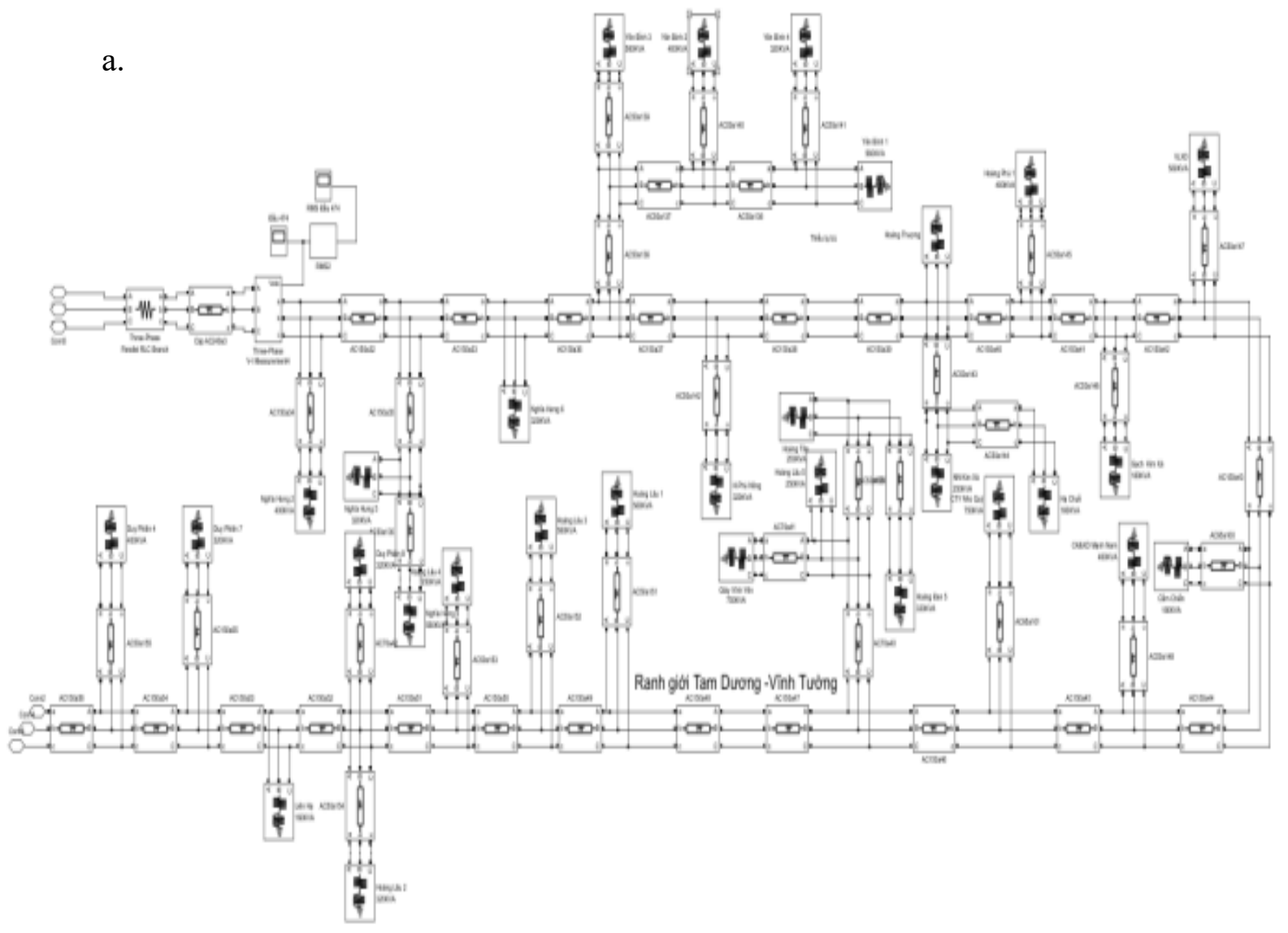

b.

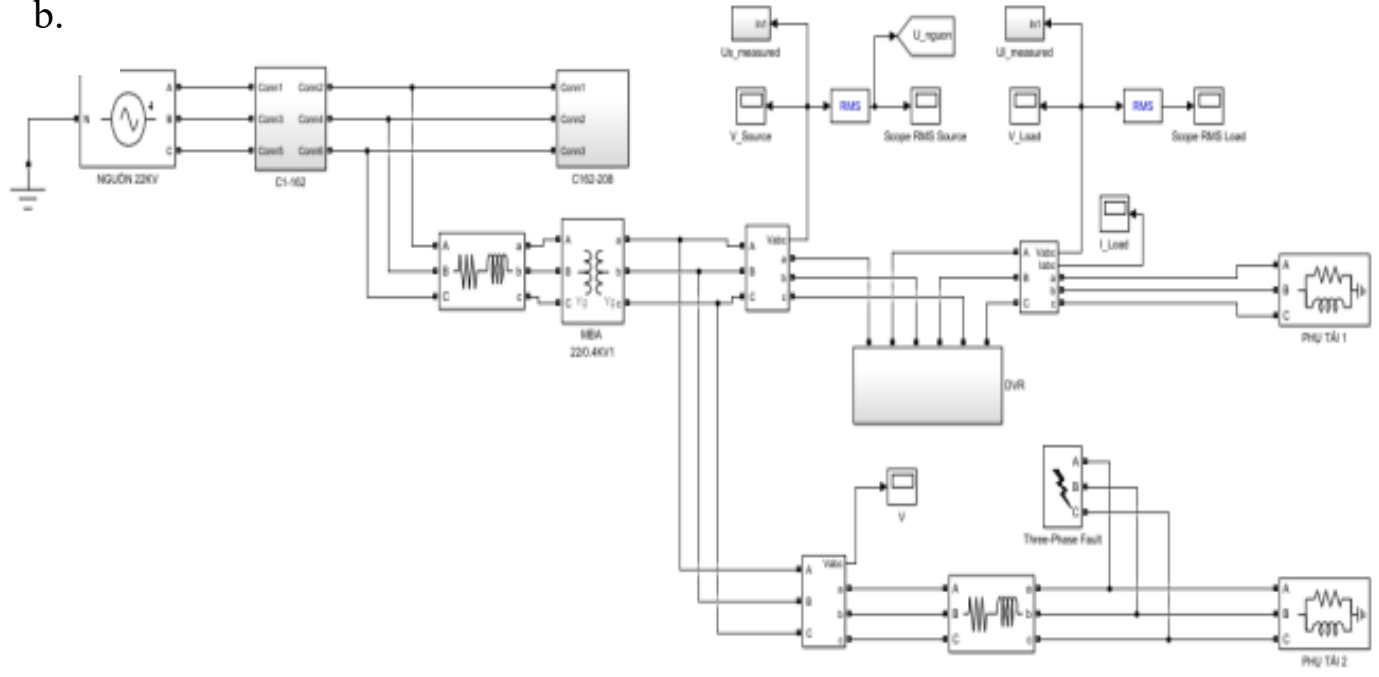

Hình 13. a. Sơ đồ mô phỏng xuất tuyến 474E25.5; b. Sơ đồ tính toán xuất tuyến 474E25.5 
Tải tuyến tính : mô hình mô phỏng tải PQ có công suất là $2,27 \mathrm{kVA} ; \cos \varphi=0,9$

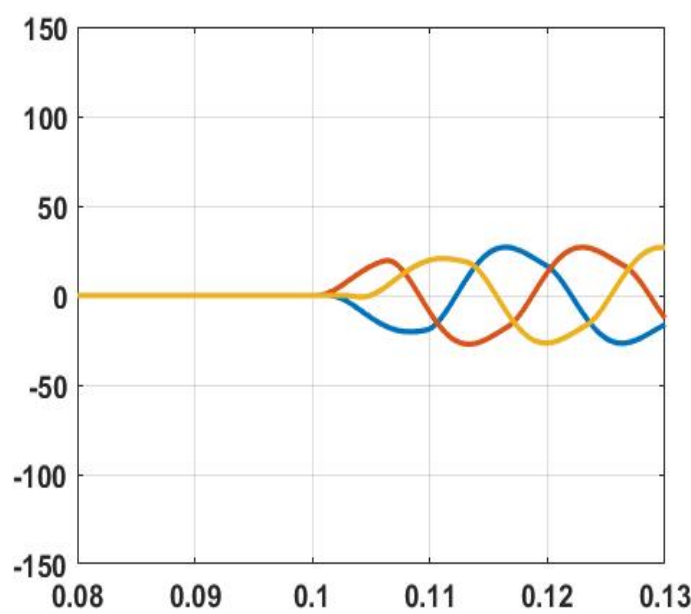

a.

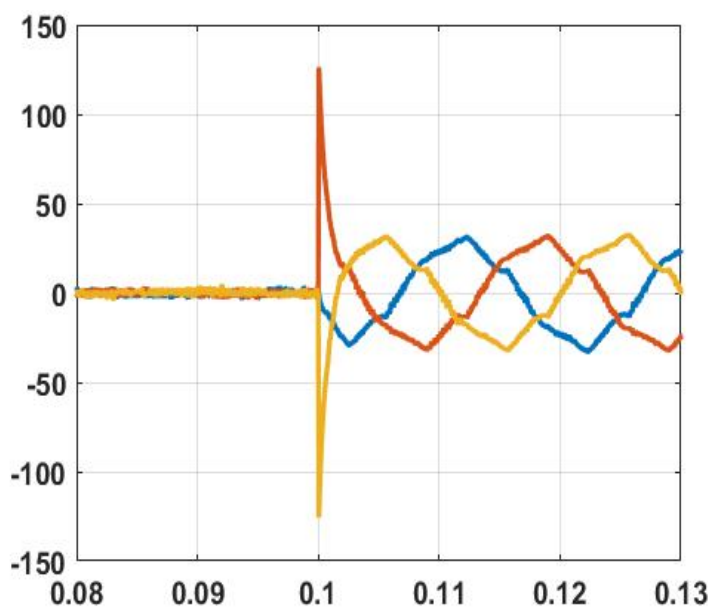

b.

Hình14. a. Điện áp bù DVR sử dụng phương pháp LES; b. Điện áp bù DVR sử dụng Phương pháp Clark-Park
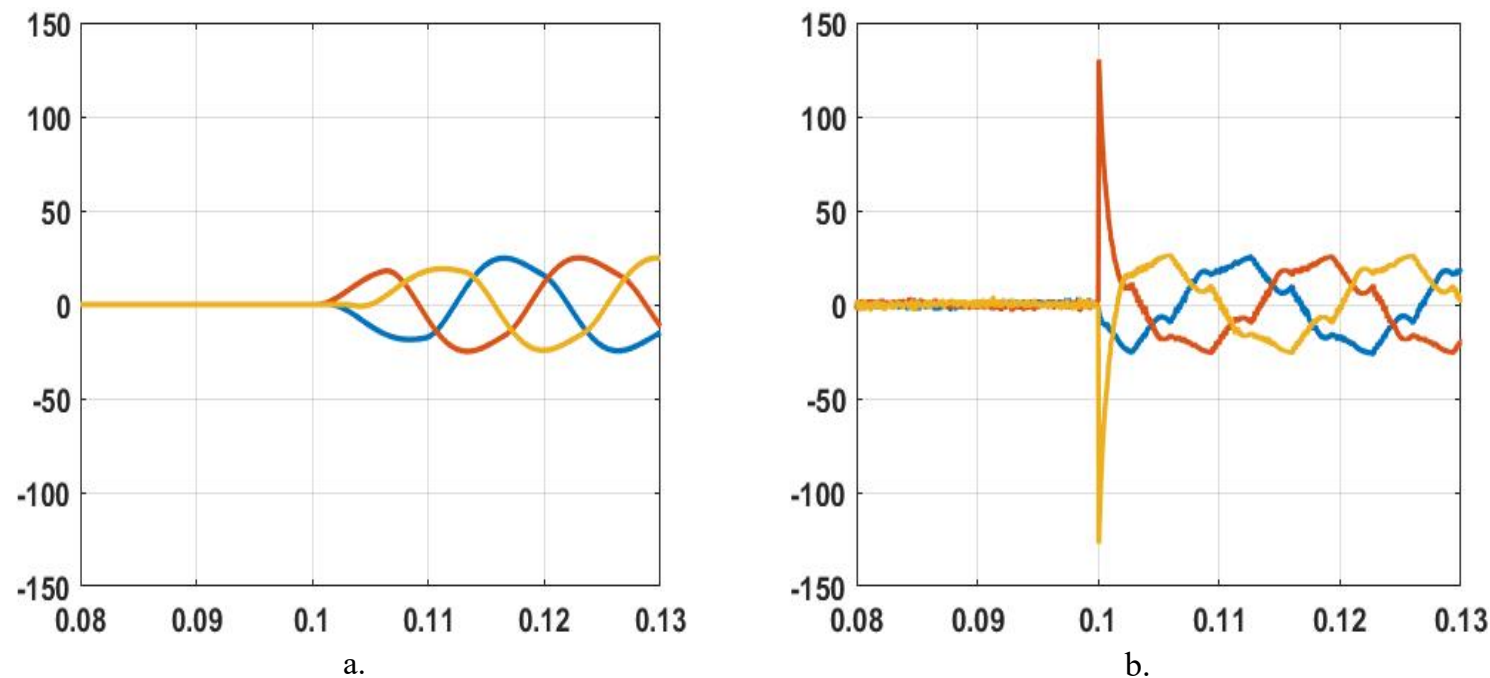

Hình 15.a. Điện áp bù DVR sử dụng phương pháp LES; b. Điện áp bù DVR sử dụng phương pháp Clark-Park

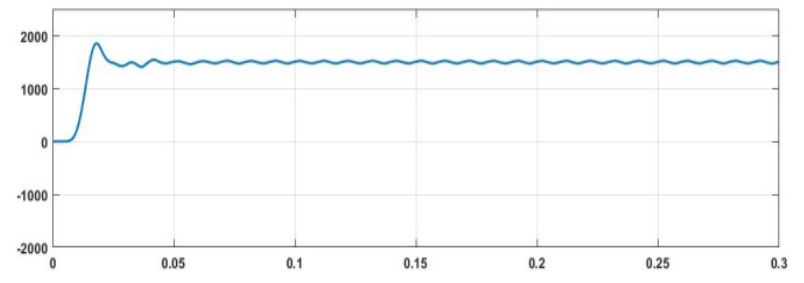

a.

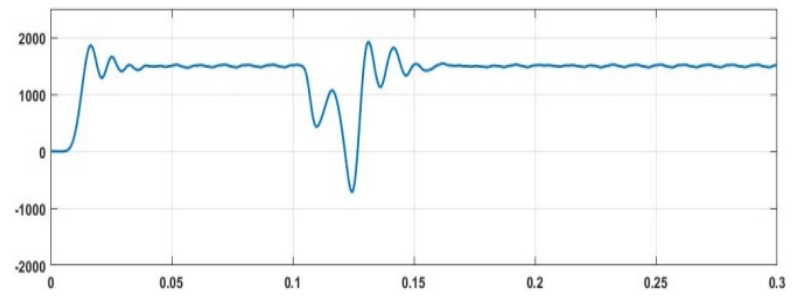

c.

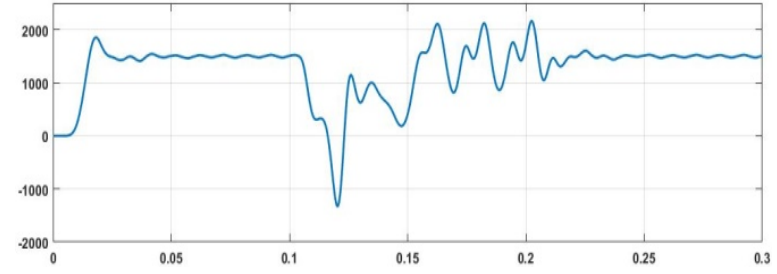

b.

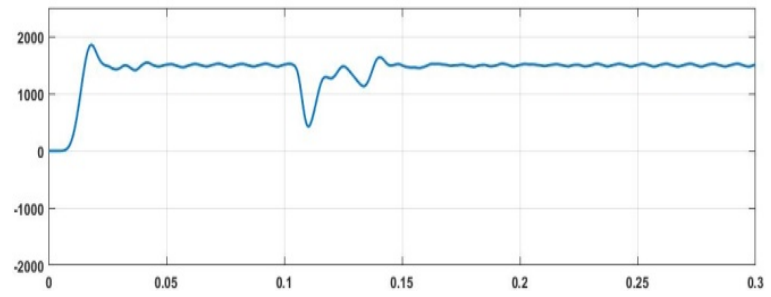

d.

Hình 16. a. Tốc độ roto lúc chưa có sự cố; b. Tốc độ roto lúc sự cố NM chưa kết nối DVR; c. Tốc độ roto lúc sự cố NM sử dụng Clark-Park; d. Tốc độ roto lúc sự cố NM khi sử dụng LES 
Ta cũng có kết quả:

$T H D=2,21 \%$ khi sủ dụng Phương pháp LES

$T H D=4,31 \%$ khi sủ dụng Phưong pháp Clark-Park

Tải phi tuyến (tải động cơ không đồng bộ) có công suất là $2,27 \mathrm{kVA}$.

Ta cũng có kết quả :

$+T H D=2,23 \%$ khi sủ dụng phưong pháp LES

$+T H D=4,97 \%$ khi sủ dụng Phương pháp Clark-Park.

Hơn thế nữa, ứng dụng phương pháp đề xuất cho thấy mức độ giảm thiểu ảnh hưởng khi sự cố tốt hơn nhiều so với phương pháp truyền thống Clark-Park. Kết quả so sánh giữa phương pháp đề xuất và phương pháp truyền thống Clark-Park như sau:

Bảng 1. Kết quả mô phỏng sự cố ngắn mạch 3 pha.

\begin{tabular}{|c|c|c|c|c|c|c|}
\hline $\begin{array}{l}\text { Chỉ } \\
\text { tiêu }\end{array}$ & \multicolumn{2}{|c|}{ THD } & \multicolumn{2}{|c|}{$\begin{array}{l}\text { Sai lệch điện } \\
\text { áp bù tại thời } \\
\text { điểm sụt áp }\end{array}$} & \multirow{2}{*}{$\begin{array}{c}\text { Thời } \\
\text { gian } \\
\text { phát } \\
\text { hiện sự } \\
\text { cố }\end{array}$} & \multirow{2}{*}{$\begin{array}{l}\text { Sóng } \\
\text { hài, } \\
\text { thành } \\
\text { phần } \\
\text { DC }\end{array}$} \\
\hline PP & $\begin{array}{l}\text { Tải } \\
\text { tuyến } \\
\text { tính }\end{array}$ & $\begin{array}{l}\text { Tải } \\
\text { phi } \\
\text { tuyến }\end{array}$ & $\begin{array}{l}\text { Tải } \\
\text { tuyến } \\
\text { tính }\end{array}$ & $\begin{array}{c}\text { Tải } \\
\text { phi } \\
\text { tuyến }\end{array}$ & & \\
\hline LES & $\begin{array}{c}2,25 \\
\%\end{array}$ & $\begin{array}{c}2,23 \\
\%\end{array}$ & $\begin{array}{l}13 \\
V\end{array}$ & $\begin{array}{l}14 \\
V\end{array}$ & $\begin{array}{l}2,2 \\
\mathrm{~ms}\end{array}$ & $\begin{array}{c}\text { Không } \\
\text { có }\end{array}$ \\
\hline $\begin{array}{c}\text { Clark \& } \\
\text { Park }\end{array}$ & $\begin{array}{c}4,55 \\
\%\end{array}$ & $\begin{array}{c}4,97 \\
\%\end{array}$ & $\begin{array}{c}105 \\
\mathrm{~V}\end{array}$ & $\begin{array}{c}109 \\
\mathrm{~V}\end{array}$ & $\begin{array}{c}5 \\
\mathrm{~ms}\end{array}$ & Có \\
\hline
\end{tabular}

\section{Kết luận}

Trong phạm vi nghiên cứu của bài báo, nhóm tác giả đã nghiên cứu phương pháp ước lượng nhanh và chính xác điện áp sụt giảm ngắn hạn, nhằm ứng dụng cho bộ điều khiển thiết bị DVR, ta có thể khẳng định phương pháp sử dụng mang lại những hiệu quả cho thiết bị như sau:
- $\quad$ Phát hiện sụt áp nhanh chóng, chính xác;

- Giảm thiểu tối đa nhảy vọt điện áp, sai số góc pha;

- Cải thiện chất lượng điện áp: biên độ, THD,...

- Không chịu ảnh hưởng bởi các sóng hài tần số bậc cao, nhiễu điện áp,...

Các tính toán mô phỏng đã khẳng định tính đúng đắn của phương pháp về mặt lý thuyết và hiệu quả ngăn ngừa sụt giảm điện áp. Kết quả bước đầu này của đề tài có thể ứng dụng cho các bài toán liên quan đến vận hành lưới điện và có thể sử dụng cho bộ điều khiển của thiết bị khôi phục điện áp động DVR.

\section{Tài liệu tham khảo}

[1] Gabriele D'Antona, The Full Least-Squares Method, in IEEE Trans. Inst. Mea, vol. 52, no. 1, february 2003. https://doi.org/10.1109/TIM.2003.809489

[2] M.S. Sachdev, FIEE M.M. Giray, A Least error squares technique for determining power system frequency, IEEE Trans. Pow. Appa. Sys, Vol. PAS-98, No.6 Nov./Dec. 1979

[3] M.S. Sachdev, Senior Member, M.A. Baribeau, A new algorithm for digital impedance relays, IEEE Trans. Pow. Appa. Sys, Vol. PAS-98, No.6 Nov./Dec. 1979 https://doi.org/10.1109/TPAS.1979.319422

[4] Guo, Hengdao, Frequency tracking and phasor estimation using least squares and total least squares algorithms (2014). Theses and Dissertations-Electrical and Computer Engineering. Paper 57. https://doi.org/10.1007/978-94-017-3552-0_6

[5] P. Harichandana, M. Tech Scholar, Madhavi Sunkara, M. Tech, Improvement of DVR performance using fast and effective control scheme under various loads, ISSN: 2248-9622, Vol. 3, Issue 5, Sep-Oct 2013, pp.1807-1813

[6] Control and testing of a Dynamic Voltage Restorer (DVR) at medium voltage level, IEEE Trans. Pow. Appa. Sys, Vol. 19, no. 3, May 2004. 